\title{
Simple explanation for the reentrant magnetic phase transition in $\mathrm{Pr}_{0.5} \mathrm{Sr}_{0.41} \mathrm{Ca}_{0.09} \mathrm{MnO}_{3}$ perovskite
}

\author{
B T CONG*, P N A HUY, N H LONG and D D LONG \\ Faculty of Physics, Hanoi University of Science, 334 Nguyen Trai Street, Hanoi, Vietnam
}

\begin{abstract}
The reentrant magnetic phase transition in $\mathrm{Pr}_{0.5} \mathrm{Sr}_{0.41} \mathrm{Ca}_{0.09} \mathrm{MnO}_{3}$ perovskite is explained using the Ising spin model on the square lattice with mixed ferromagnetic and antiferromagnetic exchange interactions. It is shown using numerical calculations that this effect is strongly affected by the external magnetic field and lattice disorder.
\end{abstract}

Keywords. Perovskite; phase transition; Ising model.

\section{Introduction}

Recently the perovskite compounds, $\mathrm{A}_{1-y} \mathrm{~A}^{\prime}{ }_{y} \mathrm{MnO}_{3}$ (where $\mathrm{A}=\mathrm{La}, \mathrm{Pr}, \mathrm{Nd} . .$. rare earth metals; $\left.\mathrm{A}^{\prime}=\mathrm{Sr}, \mathrm{Ba}, \mathrm{Ca} . ..\right)$ have induced a lot of research activity with their interesting properties such as colossal magnetoresistance, charge ordering, spin glass, etc (see Rao and Raveau 1998; Coey et al 1999). Here, we concentrate on the reentrant magnetic phase transition (RMPT) occurring in $\mathrm{Pr}_{0.5} \mathrm{Sr}_{0.41} \mathrm{Ca}_{0.09} \mathrm{MnO}_{3}$. However, RMPT does occur in other compounds having composition close to $\mathrm{A}_{0.5} \mathrm{~A}_{0.5}^{\prime} \mathrm{MnO}_{3}$. In RMPT phenomenon, the ferromagnetic long range order exists in certain temperature range above $100 \mathrm{~K}$. Figure 1 presents the experimental curves on the temperature dependence of magnetization of $\mathrm{Pr}_{0.5} \mathrm{Sr}_{0.41} \mathrm{Ca}_{0.09} \mathrm{MnO}_{3}$ (Wolfman et al 1996; see also Rao and Raveau 1998). It is shown in figure 1 that the ferromagnetic order occurs in the finite temperature interval; the temperature range of FM order and absolute value magnetization, increased with the increasing field strength. The origin of RMPT is competition between two opposites: (ferromagnetic (FM) and antiferromagnetic (AF)) interactions present in materials. Magnetic manganese ions in $\mathrm{A}_{1-y} \mathrm{~A}_{y}^{\prime} \mathrm{MnO}_{3}$ perovskite have two possible valences: $\mathrm{Mn}^{+3}$ (electron configuration $3 d^{4}$ or $\left.t_{2 g}^{3} e_{g}^{1}\right)$ or $\mathrm{Mn}^{+4}\left(3 d^{3}\right.$ or $\left.t_{2 g}^{3} e_{g}^{0}\right) . t_{2 g}^{3}$ electrons are considered as localized spin $(S=3 / 2)$ and $e_{g}^{1}$ state is itinerant because its strong hybridization with $\mathrm{O} 2 p$ orbital. Magnetic order in this substituted perovskite is mainly determined by the interacting localized spins. It is well accepted that there are ferromagnetic double exchange (DE) interaction between $\mathrm{Mn}^{+3(+4)}-\mathrm{Mn}^{+4(+3)}$ pairs and a weaker antiferromagnetic superexchange in the $\mathrm{Mn}^{+3(+4)}$ $\mathrm{Mn}^{+3(+4)}$ bonds. If the distribution of $\mathrm{Mn}^{+3}, \mathrm{Mn}^{+4}$ over magnetic lattice sites can be considered as random, this problem is closely related to the one with random inter-

*Author for correspondence action (FM or AF) between spins on the lattice. Probability of each pair-interaction depends on the ratio of the concentrations, $\mathrm{Mn}^{+4}$ and $\mathrm{Mn}^{+3}$. In this contribution, we want to reformulate and apply the theory developed in our previous study on Ising model (Cong 1992) for explaining RMPT. We believe this is a simplest approach to understand the main physics of the problem although there is also a lot of work about Ising model (see for example, Coutinho-Filho and Rezende 1990).

\section{Ising model and application}

Hamiltonian of Ising model with random nearest neighbour exchanges is written as:

$$
H=-\frac{1}{2} \sum_{i j} J_{i j} S_{i} S_{j}-g \mu \bar{h} \sum_{j} S_{j},
$$

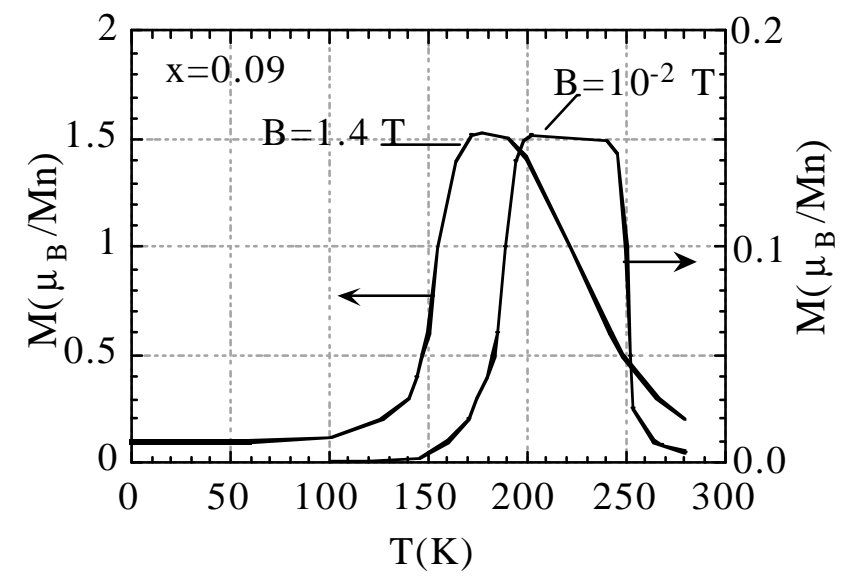

Figure 1. Reentrant magnetic phase transition (RMPT) of perovskite $\mathrm{Pr}_{0.5} \mathrm{Sr}_{0.41} \mathrm{Ca}_{0.09} \mathrm{MnO}_{3}$ in the external magnetic field (Wolfman et al 1996). 
where $\bar{h}$ denotes the external magnetic field and the first summation is taken over the nearest neighbour magnetic sites. Here the exchange interaction, $J_{i j}$, is considered as random variable and obeying proposed distribution law

$$
\begin{aligned}
& P\left(J_{i j}\right)=p \delta\left[J_{j i}-J_{\mathrm{FM}}\right]+(1-p) \delta\left[J_{i j}-J_{\mathrm{AF}}\right], \\
& J_{\mathrm{FM}}=J(1+\Delta) ; \quad J_{\mathrm{AF}}=J(1-\Delta) ; \quad \Delta>1 .
\end{aligned}
$$

In other words, $J_{i j}$ is ferromagnetic, $J_{\mathrm{FM}}$, with a probability, $p$ and antiferromagnetic, $J_{\mathrm{AF}}$, with a probability, $(1-p) . \quad J, \Delta$ are average exchange and a measure of fluctuation, respectively. The idea in many of the studies, including ours on disordered Ising model was to obtain the equation of state of the magnetic system in the algebraic form using Callen identities

$$
\begin{aligned}
& \left\langle\left\langle S_{k}\right\rangle\right\rangle_{r}=\left\langle\left\langle B_{s}\left(\beta E_{k}\right)\right\rangle\right\rangle_{r}, \\
& E_{k}=-\sum_{j} J_{k j} S_{j}-g \mu \bar{h} S_{k} ; \beta^{-1}=k_{\mathrm{B}} T,
\end{aligned}
$$

where $B_{s}(x)$ is the Brillouin function (odd function of the variable, $x$ )

$$
B_{s}(x)=\left(1+\frac{1}{2 S}\right) \operatorname{cth}\left(1+\frac{1}{2 S}\right) x-\frac{1}{2 S} \operatorname{cth}\left(\frac{x}{2 S}\right) .
$$

The inner brackets in (4) mean thermodynamic average with Ising Hamiltonian, $H$ and the outer one mean the random average with distribution function, $P\left(J_{i j}\right)$ :

$$
\begin{aligned}
& \langle\ldots\rangle=\operatorname{Tr}\left(e^{-\beta H} \ldots\right) / \operatorname{Tr}\left(e^{-\beta H}\right), \\
& \left\langle L\left(J_{i j}\right)\right\rangle_{r}=\int p\left(J_{i j}\right) L\left(J_{i j}\right) \mathrm{d} J_{i j} .
\end{aligned}
$$

Fourier transform for the right-hand side of (4) leads to the equation for on site average magnetization

$$
\begin{aligned}
& m=\left\langle\left\langle S_{k}\right\rangle\right\rangle_{r} \int_{0}^{\infty} F_{s}(t) \operatorname{Im}\left\langle\left\langle\exp \left(i E_{k} t\right)\right\rangle\right\rangle_{r} \mathrm{~d} t, \\
& F_{s}(t)=\frac{2}{\pi} \int_{0}^{\infty} B_{s}(x) \sin (t x) \mathrm{d} x .
\end{aligned}
$$

It easily shows that

$$
F_{s}(t)=\frac{\operatorname{sh}\left(\frac{2 S^{2} \pi t}{2 S+1}\right)}{\operatorname{sh}\left(\frac{S \pi t}{2 S+1}\right) \operatorname{sh}(S \pi)} .
$$

For $S=1 / 2$ we have the integral transformation (Cong 1995)

$$
B_{1 / 2}(x)=\tan h(x)=\int_{0}^{\infty} \frac{\sin x t \mathrm{~d} t}{\operatorname{sh} \frac{\pi t}{2}} .
$$

For spin one case, $S=1$, it follows from (11) that

$$
B_{1}(x)=\frac{2 \operatorname{sh}(x)}{2 \operatorname{sh}(x)+1}=\frac{4}{\pi} \int_{0}^{\infty} \frac{\operatorname{ch} \frac{\pi t}{3}}{\operatorname{sh} \pi t} \sin (t x) \mathrm{d} t .
$$

The Fourier transform method used here is different from the famous differential operator technique developed by Kaneyoshi and his coworkers (see for example, Kaneyoshi 1992). The integral transformation for higher spin order (for example $\left\langle\left\langle S_{j}^{2}\right\rangle\right\rangle$ ) can also be done. With an attempt to apply the theory for qualitative explanation of RMPT of perovskite, we use the simple case, $S=1 / 2$ ( $S_{j}= \pm 1$ in calculation), then only (9) is needed. The average in (9) is written as

$$
\left\langle\left\langle\exp \left(i E_{k} t\right)\right\rangle\right\rangle_{r}=\exp (-i \operatorname{tg} \boldsymbol{\mu} \bar{h})\left\langle\left\langle\exp \left(-i t \sum_{j} J_{k j} S_{j}\right)\right\rangle\right\rangle_{r}
$$

By expanding the exponential with Ising variables in a polynomial form we get the equation for site magnetization as follows

$$
m=\sum_{n=0}^{z} A_{n} \sum_{j_{1} \ldots j_{n}}^{z}\left\langle\left\langle S_{j_{1}} S_{j_{2}} \ldots S_{j_{n}}\right\rangle\right\rangle_{r} .
$$

The right side is sum of the correlation functions between different $n$ spins. The coefficient, $A_{n}$ in (15) is defined as

$$
\begin{aligned}
& A_{n}=\int_{0}^{\infty} \frac{a^{z-n}(x) b^{n}(x)}{\operatorname{sh}\left(\frac{\pi x}{2}\right)} \sin \left(\alpha h x+\frac{n \pi}{2}\right) \mathrm{d} x, \\
& a(x)=p \cos \alpha(1+\Delta) x+(1-p) \cos \alpha(1-\Delta) x, \\
& b(x)=p \sin \alpha(1+\Delta) x+(1-p) \sin \alpha(1-\Delta) x,
\end{aligned}
$$

where the following dimensionless quantities were introduced

$$
\beta J=\alpha ; \quad \beta g \mu_{\mathrm{B}} \bar{h}=\alpha h \text { with } h=\frac{g \mu_{\mathrm{B}} \bar{h}}{J} .
$$

In the effective field theory (EFT), which is equivalent to the Orstein-Zernik approximation, (15) for average magnetization, $m$, reduces to algebraic one for $m$

$$
m=\sum_{n=0}^{z} C_{z}^{n} A_{n}(\boldsymbol{\alpha}, p, \Delta, z, h) m^{n},
$$


where $C_{z}^{n}$ is binomial coefficient. The phase transition temperature is determined from the following equation when $h=0$

$$
1-z A_{1}\left(\alpha_{\mathrm{c}}, p, \Delta, z, 0\right)=0, \quad \alpha_{\mathrm{c}}=\frac{J}{k_{\mathrm{b}} T_{\mathrm{c}}} .
$$

For $\Delta>1$ this equation gives two phase transition temperatures: low $T_{\mathrm{C} 1}$ and high $T_{\mathrm{C} 2}$, implying an RMPT. Figure 2 illustrates the dependence of phase transition

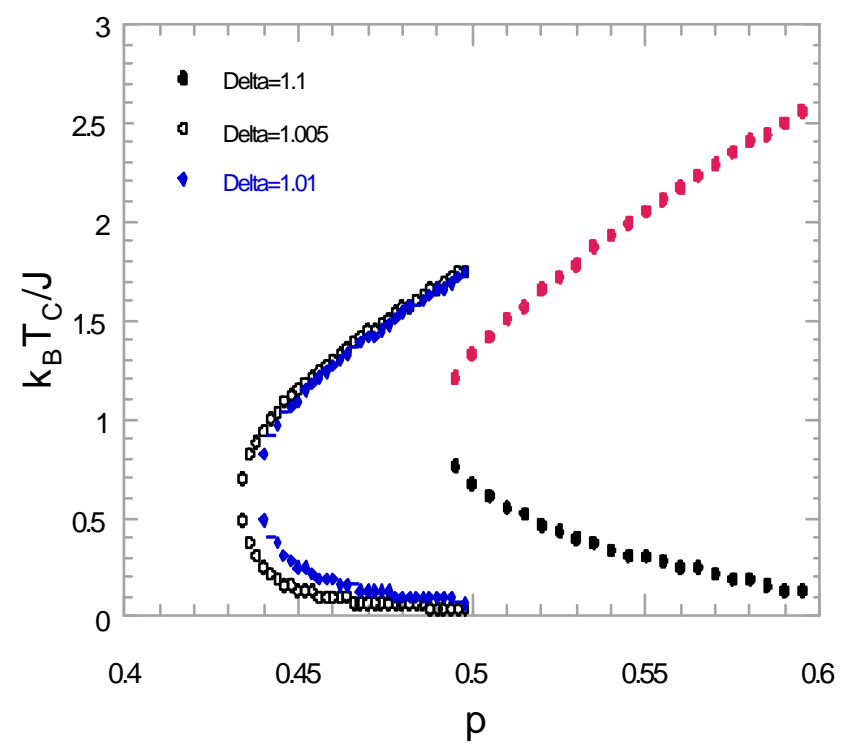

Figure 2. Dependence of RMPT on the probability of ferromagnetic interaction $p . p$ is related to the amount of divalent atom $\mathrm{A}^{\prime}$ or ratio of concentration of ion $\mathrm{Mn}^{+4}$ and $\mathrm{Mn}^{+3}$ in perovskite $\mathrm{A}_{1-y} \mathrm{~A}_{y}^{\prime} \mathrm{MnO}_{3}$. Here $z=4$ corresponds to square lattice.

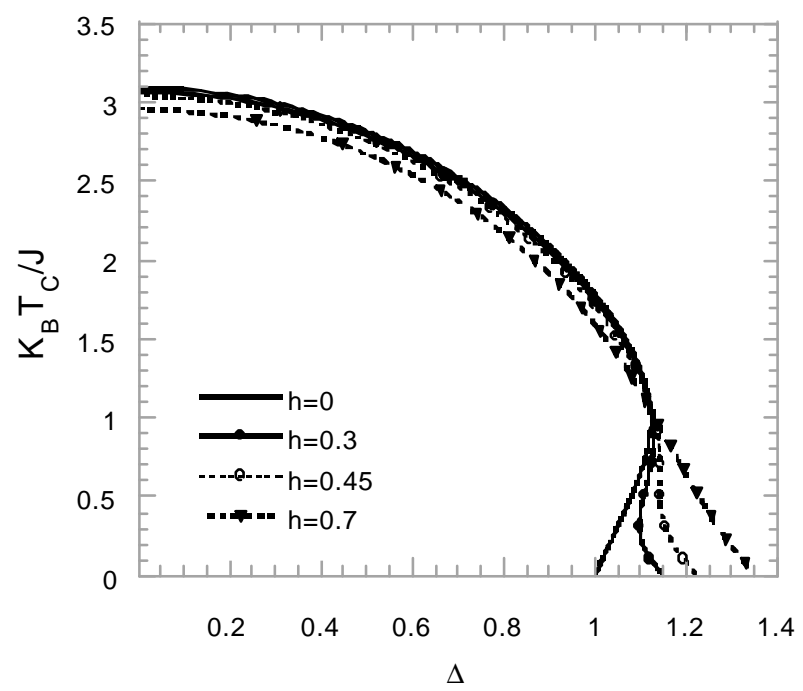

Figure 3. Destroying of RMPT by increasing external field, $z=4$.

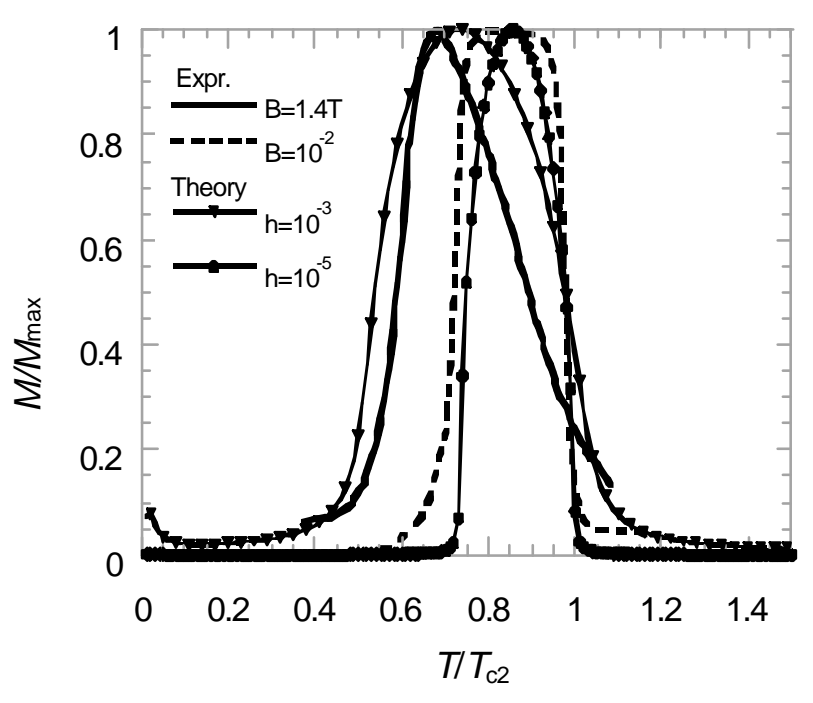

Figure 4. Comparison between theory and experiment for $\operatorname{Pr}_{0.5} \mathrm{Sr}_{0.41} \mathrm{Ca}_{0.09} \mathrm{MnO}_{3}$. For both the theoretical curves $p=0.515$; $z=4$ and $\Delta=1.138$ when $h=10^{-5}, \Delta=1.125$ when $h=10^{-3}$.

temperature on fluctuation probability of ferromagnetic interaction, $p$. For increasing fluctuation, $\Delta$, the RMPT happens for larger probability, $p$. Parameter $p$ is related to the substitution level of trivalent atoms $\mathrm{A}$ by divalent atoms $\mathrm{A}^{\prime}$ in perovskite $\mathrm{A}_{1-y} \mathrm{~A}^{\prime}{ }_{y} \mathrm{MnO}_{3}$. Here we took $z=4$ corresponding to the square plan magnetic lattice. Figure 3 shows the effect of destroying of RMPT by increasing external field. In experiment, it corresponds to the extending of $\mathrm{FM}$ region by collapsing $\mathrm{AF}$ one when external field is applied. Figure 4 gives the theoretical calculation for the experimental curves of $\operatorname{Pr}_{0.5} \mathrm{Sr}_{0.41} \mathrm{Ca}_{0.09} \mathrm{MnO}_{3}$ plotted in figure 1. For both the theoretical curves we took $p=0.515$ with a suggestion that concentrations of $\mathrm{Mn}^{+3}$ and $\mathrm{Mn}^{+4}$ are nearly equal, and $\Delta=1.138$ when $h=10^{-5}, \Delta=1.125$ when $h=10^{-3}$. This corresponds to the little increase in the effective FM exchange, $J_{\mathrm{FM}}$, in increasing field. The curves are plotted in relative dimensionless quantities, $M / M_{\max }, T / T_{\mathrm{C} 2}$ for better view. From these results, we find the average strength of $\mathrm{AF}$ (FM) exchange interaction in $\operatorname{Pr}_{0.5} \mathrm{Sr}_{0.41} \mathrm{Ca}_{0.09} \mathrm{MnO}_{3}$ to be: $J_{\mathrm{AF}}=J(1-\Delta)=2 \cdot 2 \mathrm{meV}, \quad J_{\mathrm{FM}}=J(1+\Delta)=34 \mathrm{meV} \quad$ when $h=10^{-5}$.

\section{Conclusions}

The RMPT in $\operatorname{Pr}_{0.5} \mathrm{Sr}_{0.41} \mathrm{Ca}_{0.09} \mathrm{MnO}_{3}$ is explained qualitatively by using Ising spin model in square lattice with random $\mathrm{FM}, \mathrm{AF}$ exchange interactions. One can conclude that the competition between FM, DE and AF superexchange interactions with almost equal probability in this compound is the possible reason for this phenomenon. The estimation shows that the strength of DE is about 15 times larger than the AF superexchange. 


\section{Acknowledgements}

The authors thank the Vietnam Fundamental Research Project and Vietnam National University program QGTD00-01 for support.

\section{References}

Coey J M D, Viret M and von Molnar S 1999 Adv. Phys. 48 167
Cong B T 1992 JMMM 117126

Cong B T, Hieu V T and Tuan N A 1995 JMMM 140-144 259

Coutinho-Filho Mauricio D and Rezende Sergio M (eds) 1990 New trends in magnetism (Singapore: World Scientific)

Rao C N R and Raveau B (eds) 1998 Colossal magnetoresistance, charge ordering and related properties of manganese oxides (Singapore: World Scientific)

Wolfman J, Simon Ch, Hervieu M, Maignan A and Raveau B 1996 J. Solid State Chem. 123413

Kaneyoshi T 1992 Physica A186 495 